\title{
Bipolar (bio)electroanalysis
}

\section{Laurent Bouffier, Dodzi Zigah, Neso Sojic, Alexander Kuhn}

University of Bordeaux, ISM CNRS UMR 5255, Bordeaux INP, Site ENSCBP, 16 avenue Pey Berland, 33607, Pessac, France

\section{Laurent.Bouffier@enscbp.fr}

\section{dodzi.zigah@u-bordeaux.fr}

neso.sojic@enscbp.fr

kuhn@enscbp.fr

Keywords: Bipolar electrochemistry, Analytical chemistry, Bioanalysis, Electrochemistry

\begin{abstract}
This contribution gathers a selection of the most recent studies with respect to the use of bipolar electrochemistry in the frame of analytical chemistry. Despite the fact that the concept is not new, with several important studies dating back to the middle of the last century, completely novel and very original approaches have emerged over the last few years. This current revival illustrates that scientists still (re)discover some exciting virtues of this approach, useful in many different areas, and especially for tackeling analytical challenges in an unconventional way. In several cases, this "wireless" electrochemistry strategy enables carrying out measurements that are simply not possible with classic electrochemical approaches. This review will hopefully stimulate new ideas and trigger scientists to integrate some aspects of bipolar electrochemistry in their work in order to drive the topic into so-far unexplored and eventually completely unexpected directions.
\end{abstract}




\section{Introduction}

\subsection{General introduction}

In classic electrochemical textbooks, the majority of the content is usually referring to conventional three electrode set-ups, composed of a working, counter and reference electrode(1). The electrode which is controlled in terms of potential is the working electrode, where either oxidation or reduction reactions are taking place. In bipolar electrochemistry (BPE), oxidation and reduction reactions are occurring simultaneously on the same electrode, and, most importantly, the bipolar electrode (BE) is not directly connected to a power supply. A major advantage of BPE is the straightforward and easy to implement experimental set-up. The general strategy is to place a (semi)conducting object in a solution where an electric field is generated between two electrodes. The conducting object is by definition equipotential, but the potential gradient existing in the surrounding solution will lead to an inhomogeneous potential difference between the solution and the object, which varies along its main axis parallel to the electric field lines. The direct consequence of the resulting polarization is that one extremity of the object will be preferentially the site of oxidation processes, whereas the opposite extremity will be involved in reduction reactions. Consequently, BPE generates an asymmetric reactivity at the surface of conducting objects and therefore constitutes a method of choice for breaking the symmetry in electrochemical systems. Application of BPE to the field of electroanalysis is rather recent despite the fact that the concept is absolutely not new and several important studies date back to the middle of the last century. At that time, BPE has been proposed more in the frame of industrial devices, for example as a driving force in fluidized bed reactors.(2-4) However, the current renaissance nicely illustrates that scientists discover only now some new and exciting virtues of this approach, especially in the frame of micro- and nanotechnology. Over the last two decades, many groups all over the world were able to report completely different aspects of BPE, with unforeseen potential applications in many areas, ranging from materials science to catalysis or energy storage.(5-10) A dominating playground for scientists using this concept is currently also the application of BPE for developing new strategies in analytical chemistry, ranging from classic (bio)analytical challenges(11) to more unconventional topics such as the tracking of corrosion processes.(1214) Several very attractive features are responsible for this tendency. The intrinsic advantage of adressing with one single pair of electrodes several thousands or even millions of BEs allows for example to set up high throughput screening schemes of analytes. The low-cost design is another huge advantage which cannot be neglected. Finally, yet importantly, as for many other 
electrochemical techniques, a miniaturization of the devices is rather easy and thus an essential advantage when thinking e.g. about the integration into microfluidic systems. Consequently, numerous publications have appeared in the last few years, using this concept for the development of original and sometimes quite unconventional analytical approaches. Thus, it is the right moment to summarize in this contribution the latest achievements from the last few years in the field of wireless electroanalytical chemistry. The goal is to present BPE as an appealing alternative to a broad audience of analytical scientists, hopefully convincing them that this type of experiments often allows carrying out measurements that are simply impossible with classic electrochemical approaches. We are going to discuss specifically these latter aspects after a first short introduction into some theoretical issues.

\subsection{Theoretical aspects of bipolar electrochemistry}

In this first section, we will consider the case of a conducting object immersed in a homogeneous electrolytic solution and exposed to an electric field (applied between two socalled feeder or driving electrodes). By definition, in general the object is not directly in contact with one or both feeder electrodes (Figure 1). In the specific case of an "open configuration" the object is not obstructing the electrochemical cell, thus allowing the electrolyte to still ensure ionic contact between the two feeder electrodes.

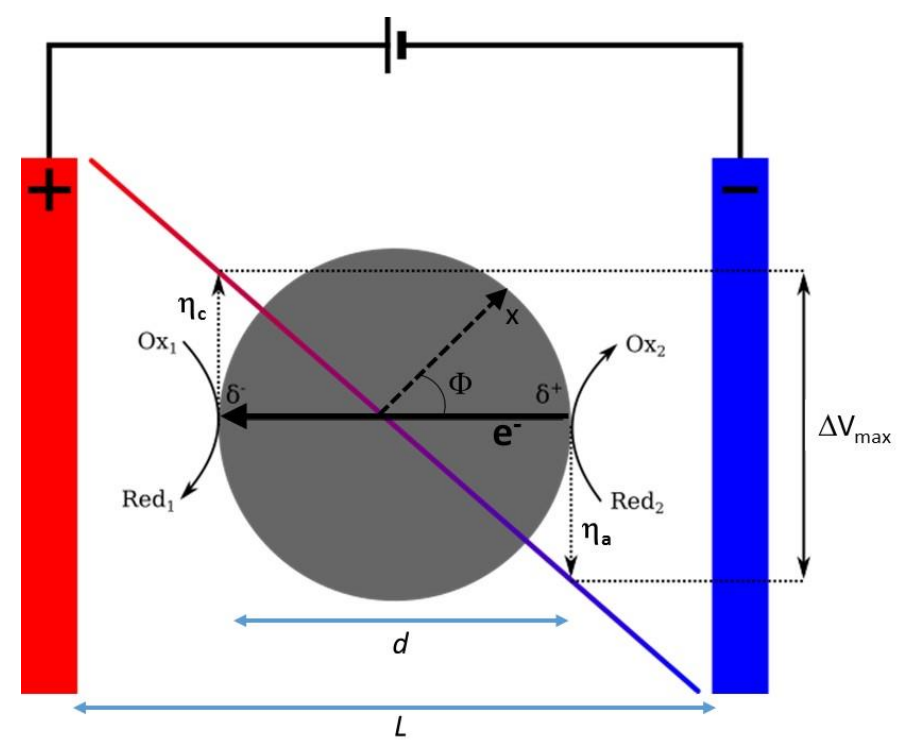

Figure 1 Scheme illustrating the polarization of a spherical conducting object with respect to the solution potential. 
When these two electrodes are biased at potentials of $V_{a}$ and $V_{c}$ respectively, an imposed potential difference $V_{\text {imp }}$ is generated between them in the solution. This gives rise to an electric field $\mathrm{E}$ in the electrolyte expressed by:

$$
\mathrm{E}=\frac{V_{a}-V_{c}}{L}
$$

with $L$ being the distance between the feeder electrodes. However, this is only a linear first order approximation (Figure 1, bicoloured diagonal line), neglecting additional potential drops which may occur at the electrode/electrolyte interfaces, as well as a distortion of the electric field by the presence of the bipolar object itself. As a result, anodic and cathodic polarization potentials $\eta_{\mathrm{a}}$ and $\eta_{\mathrm{c}}$ will be established at every position on the object. This polarization potential is a function of the localisation $x$ on the surface of the object that can be expressed as follows:

$$
\eta_{\mathrm{x}}=\mathrm{E} \frac{d}{2} \cos \Phi
$$

for a spherical object, with $d$ being the object diameter and $\Phi$ the angle between the electric field lines and a given position on the object. A maximum polarization potential difference is generated between the extremities of the object, and its value, $\eta_{\mathrm{a}}-\eta_{\mathrm{c}}=\Delta V_{\max }$, is given by:

$$
\Delta V_{\max }=\mathrm{E} d
$$

If the amplitude of $\Delta V_{\max }$ is sufficiently high, and appropriate redox active species, $\operatorname{Red}_{1}$ and $\mathrm{Ox}_{2}$, are present in solution, they can be electrochemically transformed by the following reactions:

$$
\begin{aligned}
& \operatorname{Red}_{1} \rightarrow \mathrm{Ox}_{1}+n_{1} \mathrm{e}^{-} \\
& \mathrm{Ox}_{2}+n_{2} \mathrm{e}^{-} \rightarrow \operatorname{Red}_{2}
\end{aligned}
$$

$n_{1}$ and $n_{2}$ being the number of electrons involved for each electrochemical reaction. If the two redox couples $\operatorname{Red}_{1} / \mathrm{Ox}_{1}$ and $\operatorname{Red}_{2} / \mathrm{Ox}_{2}$ have standard potentials of $E_{1}^{\circ}$ and $E_{2}^{\circ}$, respectively, then $\Delta V_{\max }$ has to be, in a first order approximation, at least equal to the difference between the two standard potentials:

$$
\Delta V_{\text {max }} \geq E_{1}^{\circ}-E_{2}^{\circ}
$$

Consequently, the conducing object behaves as a BE, promoting in a wireless way simultaneously two electrochemical reactions. Interestingly, it is possible to adjust the driving force by controlling the electric field in the solution. 
These considerations reveal that the two most important key-parameters for bipolar electrochemical reactions to occur are: $i$ ) the external electric field $\mathrm{E}$ and $i i$ ) the size of the object $d$ as indicated by Equation 3 (see Figure 1). The latter can become problematic when trying to achieve bipolar electrochemical reactions on nanosized objects. Another issue, which is intrinsically related to the open configuration, is the fact that the total current flowing through the cell, is divided into two contributions.

One fraction flows through the $\mathrm{BE}$ as a real faradaic current and triggers the bipolar electrochemical reactions. The second fraction, which can be considered as a by-pass current, flows through the solution without generating reactions at the $\mathrm{BE}$ and is strictly related to ionic flux. In order to minimize the latter one it is preferable to work with solutions of high resistance. Another option is to use a so-called "closed configuration" of the bipolar electrochemical cell. This set-up corresponds to a situation where the BE is the only existing current path, meaning that everything around the $\mathrm{BE}$ is made out of an insulator and de facto the cell is divided into two independent compartments. $(15,16)$ This has several important advantages especially for objects with dimensions in the micro- and nanometer range and also for analytical applications.(16-19) The fact that no by-pass current exists is especially interesting for cases where high current efficiencies are required. In the extreme case, almost the entire externally applied potential drop occurs between the extremities of the BE. Last-but-not-least, the cell being separated into two independent compartments, the closed configuration allows a physical separation of the reducing agents from the oxidizing ones. This might be advantage if it is mandatory to isolate reaction products from reactants for chemical compatibility reasons, or if one wants to employ different solvents in the two compartments for reasons of solubility. Each of these two configurations of BPE has its advantages and drawbacks, and therefore using one or the other will essentially depend on the specific requirements of a given analytical challenge.

\subsection{Experimental considerations}

Very different set-ups of BPE have been used over the last decades, ranging from ordinary beakers with two conducting wires acting as feeder electrodes, to microfluidic devices with specifically designed electrode patterns. The electrolytic solution can be a simple aqueous electrolyte or more exotic electrolytes such as ionic liquids. In this paragraph some general aspects will be presented, which should give the reader a brief overview and allow choosing the most appropriate cell for a given analytical task. 
BPE has quite a long history, as mentioned above. Since the early seventies, the basic concept of BPE has been used for developing different types of bipolar electrochemical cells. In this context, classic cells have been based on electrodes that are stacked between feeder electrodes in either an open or closed configuration. For the closed configuration, the absence of by-pass currents is a major advantage (vide supra). Many BEs can be found in industrial stacks(20) and their shape can vary from plates $(21,22)$ to porous conductive membranes, (23) but their use in the frame of analytical chemistry is rather limited. Bipolar cells packed with beads seem to be more interesting in this context. In this case the BEs are usually millimeter to sub-micrometer sized conducting objects, packed in an ordered or a random way. $(24,25)$ The bipolar particles should be far apart from each other to allow their efficient polarization in the bulk and avoid mutual screening effects of the electric field, as such a cross-talk would be deleterious for the efficiency of the device.

Equation 3 indicates that performing BPE becomes increasingly difficult when decreasing the size of the object, as it is necessary to apply higher electric fields to achieve the same polarization potential difference between the extremities of the BE. This is especially true for addressing micro- and nanoobjects. Theoretically, electric fields of the order of hundreds of $\mathrm{kV} / \mathrm{m}$ are then necessary, causing various practical problems such as gas bubble formation, excessive ohmic heating and massive convection in the cell. One way around this intrinsic problem is to perform BPE in a capillary electrophoresis (CE) set-up, initially proposed for analytical applications.(26) The analog set-up reported for Capillary-Assisted Bipolar ElectroDeposition (CABED)(27) was the first successful attempt to use a CE equipment for bipolar electrodeposition and constitutes also the first example of bipolar electrodeposition in the bulk of an electrolyte. CE is classically employed for separating chemicals in analytical chemistry. Usually, the capillary is inserted into a chamber where its two extremities are dipped into the electrode compartments containing the solvent. The electrodes are located outside the capillary in such a way that bubbles generated by the electrolyte decomposition cannot enter it. After being filled with a solution of interest, voltages of up to $30 \mathrm{kV}$ are applied, resulting in electric fields of up to $150 \mathrm{kV} \mathrm{m}^{-1}$ for a $20 \mathrm{~cm}$-long capillary. A UV-vis detector is located close to the outlet of the capillary, monitoring the composition of the solution leaving the capillary due to the electroosmotic flow (EOF). Performing BPE with a CE equipment has several advantages.(28) The first one is due to the high electric fields that can be applied, which is an extremely important parameter for the polarization of small objects. A second very attractive feature is the intrinsic presence of liquid flow, due to the EOF within the capillary. This flow drives continuously the bipolar objects from the inlet to the outlet. Last-but-not-least, the 
capillary as a bipolar electrochemical reactor provides a high surface area for heat dissipation and a restrictive current path which allows decreasing by-pass currents.

Although the CABED set-up allows addressing thousands and even millions of nanoparticles simultaneously in the bulk of a solution, it also has drawbacks. First of all, it uses a CE equipment that is rather expensive. Maybe the strongest restriction of the CABED approach is due to the fact that the modification takes place in a narrow capillary with an inner diameter in the range of $100 \mu \mathrm{m}$, making the process not well-adapted for scaling-up the production of modified particles for industrial applications. In order to partially overcome these limitations, capillaries with much larger diameters have been used, not only in the context of bipolar electrodeposition,(29) but also for addressing bipolar nanoobjects in the frame of analytical challenges. $(30,31)$

However, even in this case the capillary contains at most a few milliliters of solution. Therefore an alternative set-up consists in using a cell composed of one reaction compartment, in which the bipolar objects and the reagents are located, separated from the two electrode compartments by two membranes.(32) The feeder electrodes, which are immersed in the solvent containing only supporting electrolyte, are connected to a high voltage power supply. Rather inert materials such as gold, platinum or carbon are typically employed as feeder electrodes. Polymer or sintered glass membranes with satisfying mechanical stability and permeability are used as separators in order to limit the interference of bubble formation, solvent evaporation and convection, occurring in the electrode compartments when applying high electric fields, with the reaction compartment. However, one has to be aware that such separators induce an additional potential drop, and thus lower the effective electric field in the reaction compartment. In addition to the set-ups described above, a certain number of more special configurations in terms of cell design, electrodes and experimental conditions have been developed in recent years and are described in more detail in the following sections.

\section{Analytical applications}

\subsection{Analytical readout schemes}

In the last few years, different strategies have been developed to use BPE as an analytical technique. In all the configurations, the BEs has a sensing site, to detect the analyte, and a reporting site to quantify the analyte (Figure 2).(33) The simple experimental set-up is composed of a generator, connected to the anode and the cathode, to apply the electric field, and a conducting object located between the two electrodes, which will be the BE. In certain 
cases, an additional instrument to measure the signal (e.g. light) emitted from the reporting site is required. As mentioned above, the open configuration is one possibility to carry out experiments in the frame of analytical chemistry.(30) For example, an open configuration based on the use of nanopipettes has been reported. In this configuration, the inner wall of a nanopipette tip is decorated by a metallic layer, $(34,35)$ and wireless electroanalysis has been performed at the tip of the nanopipette.(36) In the case of the closed configuration several approaches have been reported, depending on the type of BE connecting the two compartments. Microchips with electrode arrays, membranes filled with conducting materials and split BEs, connecting the two independent compartments via an external wire, have been used in this context. One of the major advantages of BPE is the ability to address simultaneously a large number of objects in order to carry out multiple detections. Therefore, chips containing several BEs have been prepared by (photo)lithography in order to generate micrometer-sized electrodes allowing parallel analysis.(37-44) An interesting alternative to (photo)lithography, which is expensive, is the use of paper-based microsystems.(45-50) This approach, based on screenprinting technology, represents an affordable strategy to develop even quite sophisticated devices without using (photo)lithography. Chips can also be made by simply using an ITO slide with PDMS slices. $(51,52)$ When using a membrane to separate the sensing and the reporting compartments, the membrane channels can be filled with conducting material to create a massive array of nanoelectrodes, which will ensure the faradaic charge transfer between the two compartments.(53-55) While in most of the cases, the BE is a single object, it is also possible to connect two individual electrodes, located in the respective compartments, with a wire in order to transform them into one BE. Such an electrode is called a split-BE. The split$\mathrm{BE}$ offers the possibility to spatially separate the sensing and the reporting compartments at large distances. This method has been used to connect a carbon electrode with another carbon electrode,(56, 57) a semi-conductor,(58) or a screen-printed electrode.(59) Two microelectrodes can also be connected together.(60-62) Furthermore, a suitable configuration is also to couple a bipolar electrochemical reaction with measurements with a potentiostat.(56, 63-65)

Recently, a very original set-up without any feeder electrodes has also been proposed. The anodic and cathodic poles of the generator were directly connected to an ITO plate. The internal resistance of the ITO electrode induces a potential gradient along the electrode, and thus a variable polarization with respect to the solution which can trigger anodic and cathodic reactions at opposite locations on the surface of the plate.(66) 


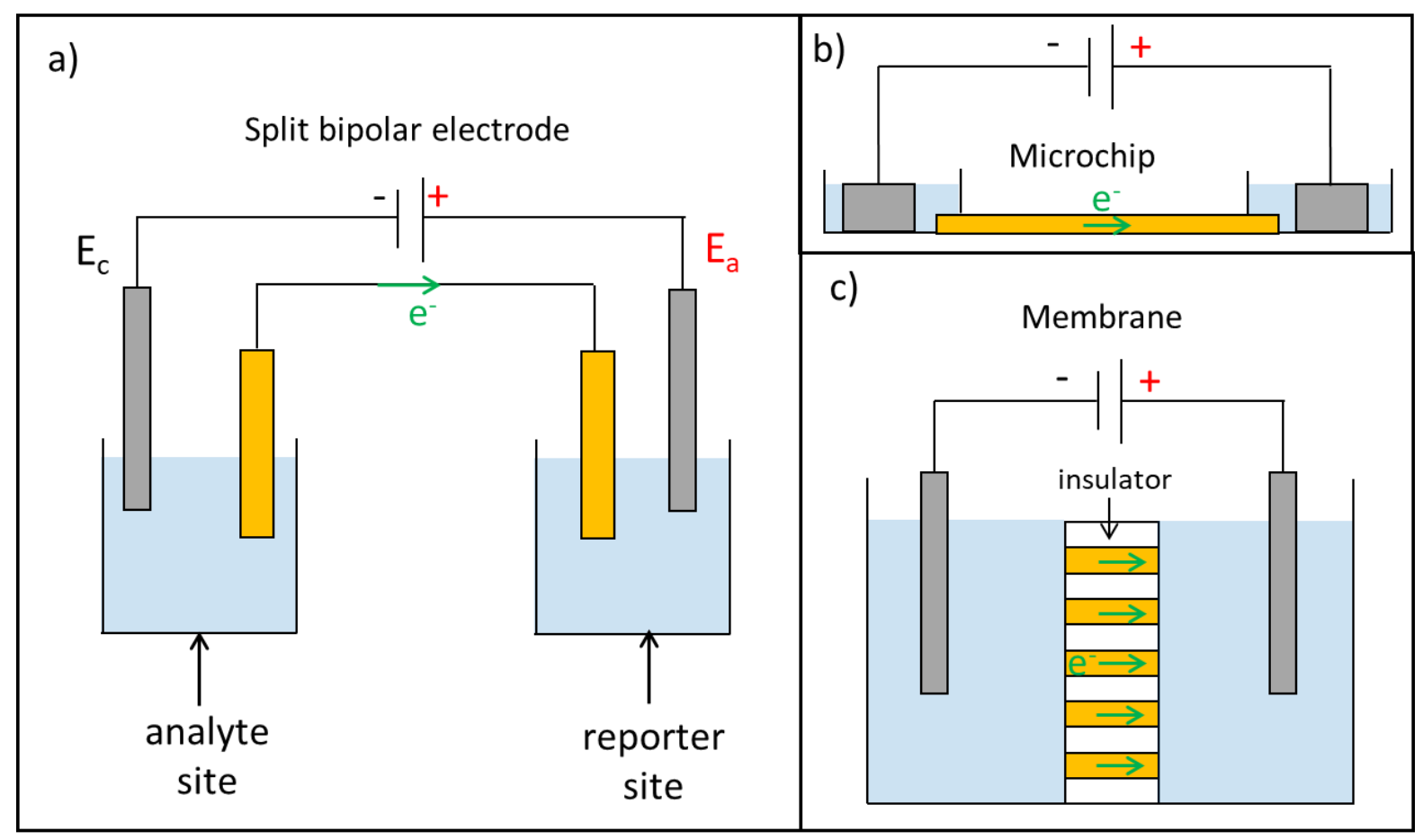

Figure 2 Schemes illustrating different possible set-ups for a closed bipolar electrochemical cell.

The majority of the reporting sites use an optical readout based on electrochemiluminescence (ECL).(38, 39, 67, 68) In order to trigger an ECL reaction, an electroactive luminophore is introduced into the solution. The bipolar electrochemical process initiates a series of chemical reactions that will trigger light emission. The two most employed ECL-active dyes are tris(2,2'bipyridine)ruthenium $\left(\left[\mathrm{Ru}(\mathrm{bpy})_{3}\right]^{2+}\right.$ ) and 3-aminophthalhydrazide (luminol). Usually, a sacrificial co-reactant has to be present in the solution in order to promote the excited state of the luminophore. Tri-n-propylamine (TPA) and hydrogen peroxide $\left(\mathrm{H}_{2} \mathrm{O}_{2}\right)$ are employed with $\left[\mathrm{Ru}(\mathrm{bpy})_{3}\right]^{2+}$ and luminol, respectively. Fluorescence emission is another type of optical readout, for example, by using a fluorogenic reaction that will convert an electrochemical signal into a fluorescent signal.(69) The redox couple resazurin/resorufin is interesting, because resazurin is a weakly emissive molecule, while resorufin is a much brighter luminophore.(70) Instead of light emission, an electrochemically-induced change of color (i.e. electrochromism) can also be employed as an optical readout. ${ }^{42}$ In this context, methyl viologen $\left(\mathrm{MV}^{2+}\right)$ is often used because the di-cation and the radical cation $\mathrm{MV}^{{ }^{+}}$are colorless and purple, respectively.(71-73) The potential induced color change of Prussian Blue is also a good alternative, as has been demonstrated for example in the frame of potentiometric sensing arrays composed of closed BEs.(74) To detect the light emitted by the reporting site, a simple digital 
camera(75) or a smartphone $(42,76)$ enables quantitative measurements. However, for a more precise monitoring, the emitted light is mainly recorded with a microscope equipped with a CCD camera $(62,77,78)$ or a photomultiplier tube (PMT). $(52,79)$ The light from the reporting site can also be guided to the camera by an optical fiber.(59, 80, 81) Non-optical readout concepts are equally available, such as potential or current measurements,(82-84) electromechanical actuation,(85-87) or the dissolution of metals.(88)

\subsection{Electroanalysis}

BPE can be used to perform various types of electroanalytical measurements, either for characterizing materials, or to detect analytes. For example, Eßmann et al. investigated the bifunctional water-splitting catalyst $\mathrm{Co}_{2} \mathrm{~B}$ for hydrogen evolution reaction (HER) and oxygen evolution reaction (OER). Using this approach, all the key parameters for both reactions (overvoltage, half-cell potential and catalyst stability) were obtained in a single experiment by using a split-BE.(82) Defnet et al. used two carbon microelectrodes as a split-BE to study transient collision events of single Pt nanoparticles. The split-BE converted the amperometric signal generated for each collision at the sensing site into an optical signal on the reporting site (Figure 3). On single Pt nanoparticles (70 nm), the fast transient HER ( 400 ms) at the cathodic side was measured via the ECL signal at the anodic side. This study opens up the way for imaging transient redox processes when large arrays of closed BEs are used.(61)

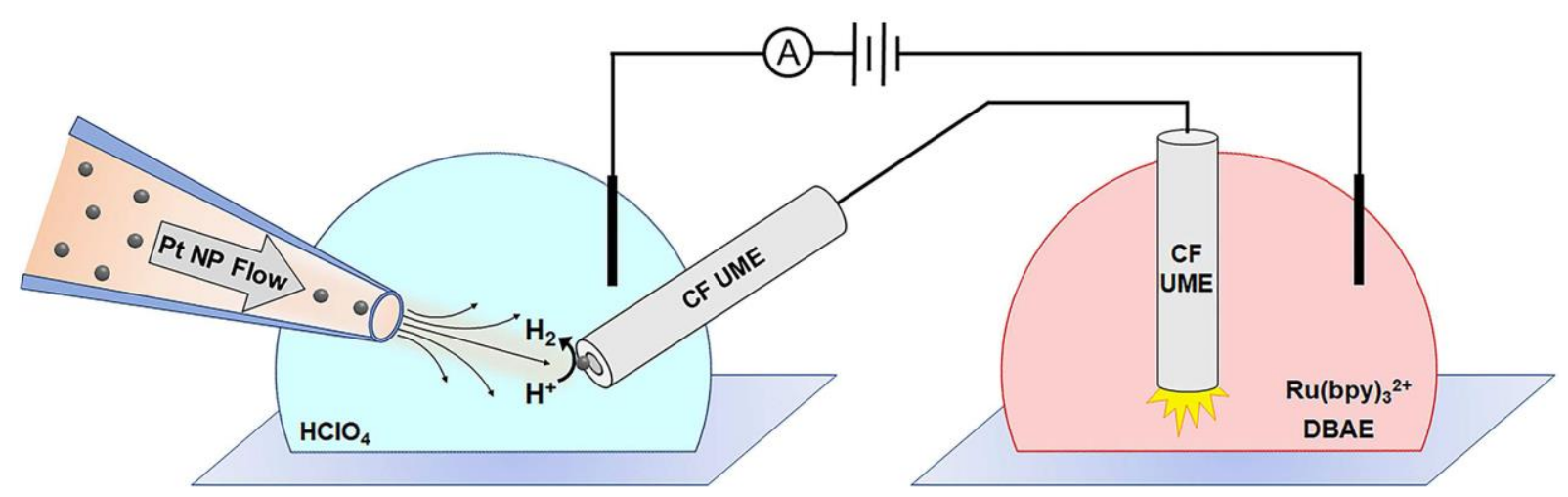

Figure 3 Scheme of the experiment coupling Pt nanoparticle collisions to anodic ECL across a split-BE composed of two carbon fiber ultramicroelectrodes (CF UME). The microjet collision and the reporting ECL were characterized on two separated inverted microscopes. Reproduced from reference(61) with permission from John Wiley and Sons. 
Han et al. demonstrated that a nanopipette $(\sim 60 \mathrm{~nm}$ in diameter) can be used to observe transient BPE on a single silver nanoparticle $(50 \mathrm{~nm})$. When the particle approaches the nanopore aperture, it is possible to oxidize one side of the particle and to produce $\mathrm{H}_{2}$ on the other side due to the focusing of the electric field. These experiments allow studying gas generation on nanocatalyst materials.(89) An original method that combines the advantages of BPE and electrochemical microscopy has been developed under the name of Scanning bipolar electrochemical microscopy by Santos et al. This recent technique employed two microelectrodes in the split-configuration, to observe small changes in local $\mathrm{O}_{2}$ concentrations at a photocatalyst $(\mathrm{Mo-BiVO}$ ) revealed by the ECL signal on the reporting site.(62)

It is also possible to detect heavy-metals $\left(\mathrm{Cu}^{2+}, \mathrm{Cd}^{2+}\right)$ in water by using potential-resolved multicolor ECL with the split-configuration based on the combination of a screen-printed electrode and a simple glassy carbon electrode.(59) BPE has also been used to develop new types of $\mathrm{H}_{2} \mathrm{O}_{2}$ sensors, either by changing the morphology of the electrode,(75) or by playing with the composition of the solution at the reporting site.(79) Using nanoporous ITO at the sensing site Seo et al. reported a linear detection range of 0.02-5 mM and a LOD of $\sim 20 \mu \mathrm{M}$ for $\mathrm{H}_{2} \mathrm{O}_{2}$. The LOD was divided by a factor of ten compared to bare ITO.(75) Hao et al. also employed allinorganic perovskite $\mathrm{Cs} \mathrm{PbBr}_{3}$ quantum dots at the reporting site to detect $\mathrm{H}_{2} \mathrm{O}_{2}$. The quantum dots emit an ECL signal at the anodic pole simultaneously with the reduction of $\mathrm{H}_{2} \mathrm{O}_{2}$ at the cathodic pole, leading to a linear detection range of 1-200 mM and a LOD of 50 $\mu$ M.(79) Ibañez et al. developed bipolar spectroelectrochemistry. They used UV-vis spectroscopy with optical fibers in the reflection mode to observe oxidation and reduction reactions on the same BE. The set-up allows separating the electrochemical signal into two different optical signals from the two compartments. The behavior of redox molecules such as $\mathrm{Ru}(\mathrm{bpy})_{3}{ }^{2+}, \mathrm{Fe}\left(\mathrm{CN}_{6}\right)^{4-}$ and $\mathrm{IrCl}_{6}{ }^{3-}$ , but also conducting polymers (EDOT) have been studied with this method. The coupling of these two techniques promises to offer interesting analytical possibilities in the future.(81) In addition to the readout schemes used above, a completely new approach appeared very recently, based on the mechanical deformation of a conducting polymer triggered by BPE. In these cases the analyte is oxidized at one extremity of a bipolar polymer strip, whereas a reduction of the conducting polymer itself occurs at the opposite side. The latter one is accompanied by an exchange of ions, which leads to shrinking or swelling of the polymer and finally to a controlled deformation of the bipolar object.(90-92) The rate and amplitude of deformation is directly correlated with the concentration of analyte and thus this approach could be successfully used to detect various analytes, ranging from $\mathrm{H}_{2} \mathrm{O}_{2}$ to glucose(85) with good linear range and detection limits. An additional aspect, which has been explored based on this detection 
approach, is the enantioselective recognition of chiral analyte molecules. When the positively polarized side of the polymer strip is post-modified with an enantioselective material, either chiral metal(86) or intrinsically chiral polymers(87), the oxidative conversion becomes stereoselective and therefore the deflection of the polymer at the $\delta^{-}$extremity will be more pronounced in the presence of one of the enantiomers, or even allows absolute chiral recognition, providing an on/off answer when one or the other of the molecular antipodes is present in solution (Figure 4).
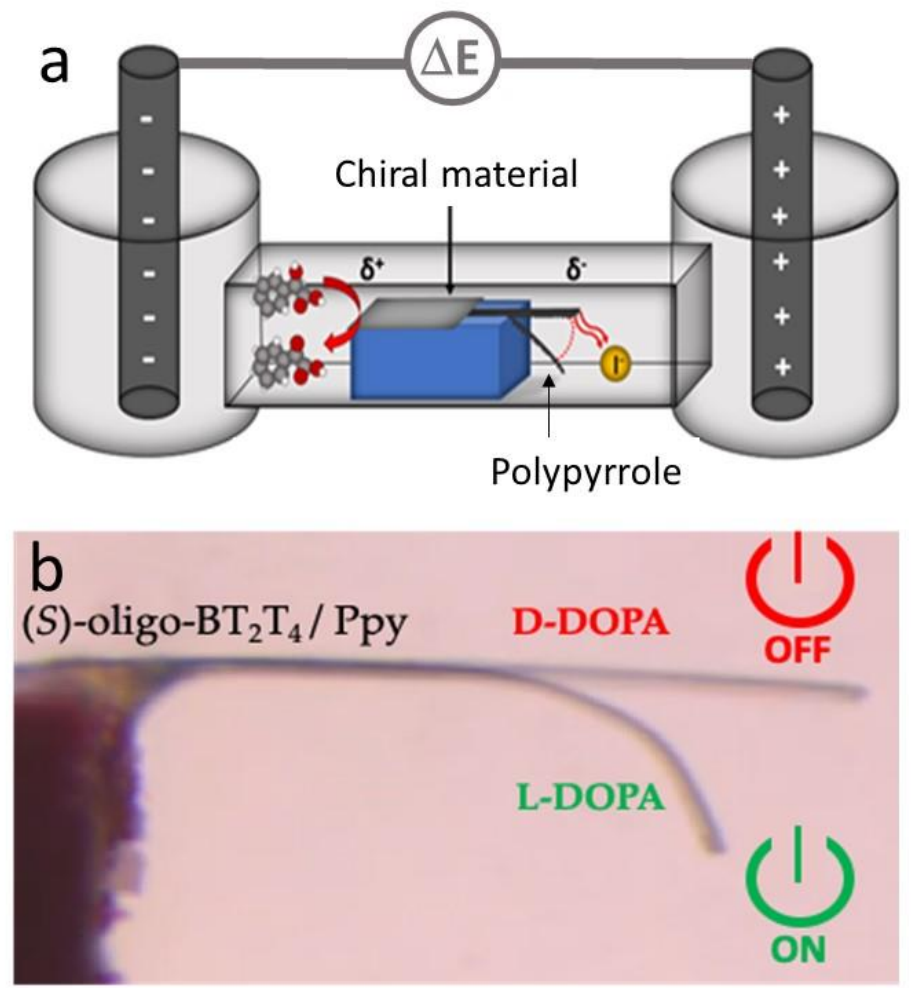

Figure 4 a) Scheme of the experimental set-up allowing a bipolar electromechanical readout of analytical information (top). Reproduced from reference (86) with permission from The Royal Society of Chemistry. b) Superposed optical microscopy pictures of the wireless bending induced on a polypyrrole strip in the presence of the wrong enantiomer (off state) or the good enantiomer (on state) when an intrinsically chiral polymer is used as a selector on the positively polarized extremity of the polymer strip (bottom). Reproduced from reference (87)with permission from the American Chemical Society. 


\subsection{Bioelectroanalysis}

\subsubsection{Enzymes}

Enzymatic systems have often been combined with BPE, mainly to develop original bioanalytical platforms exploiting the versatility of BPE. For the analytical applications, as already discussed, a major challenge associated with BPE is the readout of the signal due to its wireless feature. Since the electrochemical activity of a BPE system can be converted quantitatively into an optical signal, as reported initially by Manz,(93) the different optical readout methods discussed previously, such as ECL, fluorescence, colorimetry, electrochromism, etc. $(6,33,94)$ have been also used for bioelectroanalysis. For BPE, redox enzymes are exploited to convert target analytes into redox-active or optically active species. Bohn and coworkers described a multiplexed closed bipolar sensor for the simultaneous detection of lactate, glucose and uric acid.(95) The activity of these specific oxidases was coupled with an electrochromic reaction in a separated reporter cell in such a way that the magnitude of the color change is correlated with the concentration of the enzymatic substrates in the readout compartment. Moreover, the device was fabricated as a paper-based carbon electrode and the color change was simply recorded by a smartphone. Such a straightforward readout makes the approach very attractive and this is reinforced by the disposable character of the device. Based on the electrochromism of Prussian Blue films, a resettable and reprogrammable enzymatic keypad lock system was presented for the direct detection of ascorbic acid and glucose by the naked eye.(96)

Fluorescence based reporting reactions were also combined with BPE and enzymes. The oxidation of dihydroresorufin into resorufin, a highly fluorescent dye, was successfully applied for the detection of glucose and $\mathrm{H}_{2} \mathrm{O}_{2}$ using enzyme modified electrodes at the reporting site.(57) ECL, which is a very sensitive analytical method,(97) was also coupled with BPE in closed as well as open configurations. $(46,98,99)$ Indeed, the "by-products" of the enzymatic activity of dehydrogenases and oxidases, such as NADH and $\mathrm{H}_{2} \mathrm{O}_{2}$ may react with $\left[\mathrm{Ru}(\mathrm{bpy})_{3}\right]^{2+}$ or luminol and generate orange-red or blue ECL emission, respectively. The multiplexed detection of glucose, lactate and choline was reported using the corresponding oxidases on a $\mathrm{BE}$ array together with luminol ECL.(39)

A paper-based fluidic device operating in closed bipolar mode was fabricated by wax and a carbon ink-based screen-printing process.(100) The quantitative ECL based analysis of $\mathrm{H}_{2} \mathrm{O}_{2}$ and glucose was demonstrated in four complex samples (human serum, urine, wine, and glucose injection). The band-shaped paper-based fluidic device represents an interesting 
configuration with easy and low-cost fabrication procedures. Multicolor ECL emission has been previously reported in an open bipolar configuration for single conducting objects as well as for a dispersion of micro/nano-objects. $(31,68)$

Since BPE enables the electrochemical addressing of a multitude of conductive objects,(25) bulk ECL imaging was combined with enzymatic coreactant production for the sensitive remote detection of two different analytes, namely, choline and glucose.(30) It is noteworthy that bipolar ECL imaging was performed simultaneously with dehydrogenase-type and oxidase-type enzymes, leading to concomitant orange-red and blue ECL emissions. In addition, this original approach provides a global chemical view through one single ECL image of inhomogeneous samples such as a biochemical concentration gradient formed in a capillary.(30)

The selectivity of the enzymes has been recently combined with the confinement of the voltage drop in spatial restriction by Jiang and coworkers to perform intracellular wireless analysis of single cells by bipolar ECL.(36) As described previously in Section 2.1 and reported by several groups, the voltage drop is confined within the nanopipette tip, $(35,89,101-103)$ which generates ECL emission at very low voltage. The inner walls of a nanopipette tip were decorated by a Pt deposit that is used as an open bipolar ECL device (Figure 5). The electric field provokes the sorting of various molecules from the intracellular space into the nanopipette, where they react and produce ECL signals. The synergetic action of the nanopipette and the bipolar enzyme-based ECL approach allows measuring in vivo the intracellular concentrations of hydrogen peroxide, glucose and the intracellular sphingomyelinase activity (i.e. hydrolase) with the corresponding enzymes. This original strategy, which requires a remarkably low voltage, minimizes an eventual bias of the cellular activity and provides a novel concept in the field of single cell electrochemical analysis. 


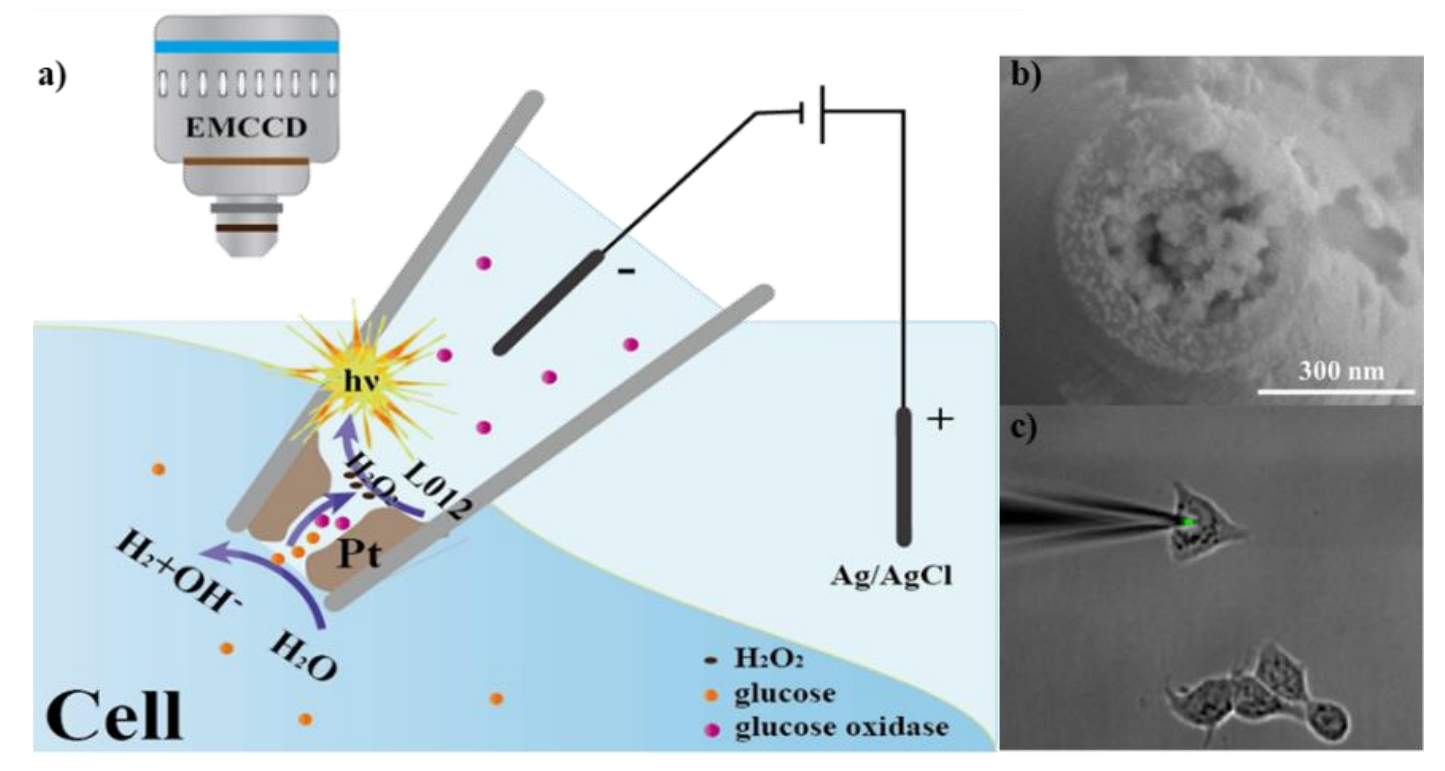

Figure 5 a) Scheme of the bipolar ECL detection at a nanopipette tip with the inner walls decorated by a Pt deposit. The tip is inserted into the cytosol for intracellular wireless analysis. b) SEM image of the Pt deposit at the nanopipette tip (front-view). c) Overlayed bright-field and ECL images of the nanopipette showing the detection of intracellular glucose in single living non-stimulated HeLa cell. The green spot originates from the ECL emission confined at the nanopipette tip.(36) Adapted with permission from John Wiley and Sons.

As described above, the majority of the enzyme-based developments using BPE are focused on bioanalytical applications. However, BPE has been also coupled with enzymatic systems to investigate enzymatic activities and construct enzymatic cells. The versatility of BPE was applied to prepare both, a biocathode (bilirubin oxidase immobilized in a thiophene-based film) and a bioanode (glucose dehydrogenase immobilized on $\mathrm{Au}$ nanostructures) for mediatorless/membraneless biofuel cells.(104) The assembled glucose $/ \mathrm{O}_{2}$ cell offers a power output of $146 \mu \mathrm{W} \mathrm{cm}{ }^{-2}$ with an open circuit voltage of $0.54 \mathrm{~V}$. It illustrates an interesting application of BPE in the field of bioenergy. Conzuelo and coworkers reported the electrical coupling of different photosystems and the resulting photobioelectrochemical cell.(105) Photosystems 1 and 2 were embedded and wired to the underlying electrode using an osmiumcomplex-modified redox polymer in a closed setup. The response upon illumination was studied. The effects of different parameters such as $\mathrm{pH}$, temperature, electron acceptor or inhibitor concentration were investigated. Such a photobioelectrochemical study of the enzymatic performances provides interesting insights into the working principle of closed bipolar systems. Another enzymatic system, cytochrome c, was also studied in a 4-electrode 
closed bipolar cell and direct electron transfer has been demonstrated between cytochrome $\mathrm{C}$ and the aqueous pole of a BE in aqueous-organic electrolyte solutions.(106) This interesting approach allows investigating electron transfer between cytochrome $\mathrm{c}$ and redox mediators at the interface between two immiscible electrolyte solutions (ITIES), which mimic soft biomembranes. The wireless feature of BPE combined with the selectivity provided by the enzymes offers a wide range of bioanalytical sensing strategies with disposable miniaturized devices.

\subsubsection{DNA}

DNA is a classic bioanalytical target in the field of biosensors. In that context, one can discriminate two classes of electrochemical sensors that use DNA as a key ingredient. The first strategy, that was historically developed, relies on the immobilization of single stranded DNA (ssDNA) at the surface of an electrode in order to use such a modified surface for the detection of the complementary sequence through DNA hybridization. On the other hand, there are many other examples where DNA is not necessarily the analytical target, even if nucleic acid recognition processes are involved. For DNA sensing, BPE offers many advantages in comparison with conventional electrochemistry: 1) Since two electrochemical reactions are coupled across the BE, it is an ideal way to separate the sensing process and the reporting reaction. 2) A large ensemble of BEs can be employed in parallel, thus allowing highthroughput screening or multiplexing. 3) The special case of the closed-configuration offers the possibility to use two different electrolyte solutions in the two half-cells that are physically separated (aqueous/organic, different chemical composition, $\mathrm{pH}$, etc.). The first example of wireless DNA sensing with ECL readout was originally proposed by Crooks and coll.(107) From that early report, the same team and others were able to imagine additional schemes for DNA detection that were already reviewed several times.(6, 33, 108, 109)

Focusing on the most recent contributions, an enhanced ECL detection strategy on a closed BE was proposed for the detection of prostate specific antigen (PSA).(110) In this report, the target is recognized on the cathode part of the BE whereas the anode serves as reporting pole. For the latter, a bottom up approach was adopted with the immobilization of the ssDNA probe prior to hybridization with the complementary strand labelled with a gold nanoparticle (Au NP). This NP catalyzes anodic ECL involving luminol and $\mathrm{H}_{2} \mathrm{O}_{2}$, offering an excellent readout signal. Another strategy that involves the same Au NP-modified DNA was reported for the detection of cancer cells.(111) As before, these Au NPs catalyze ECL, but also act as seeds for the chemical reduction of $\mathrm{Ag}^{+}$cations released from the anodic dissolution of silver. In that case, 
the formation of the silver layer surrounding the Au NPs completely quenches the ECL emission of luminol. An original approach based on a direct ohmic loss principle was also proposed for the DNA-mediated detection of thrombin.(56) The authors studied in detail the influence of key experimental parameters on the iR drop of a closed BPE device. They rationalized this effect by the peak separation of a potassium ferro-/ferricyanide redox probe, enabling a new class of biosensors by connecting sensing units with tunable resistance. The principle was exemplified with a thrombin aptamer in combination with an Au-modified complementary DNA. Again, these Au NPs were used to mediate the growth of Ag, which directly affects the resistance in the gap.

An ECL strategy for the detection of the HIV genome was also designed on a closed splitBE.(112) Anthraquinone disulfonic acid was used as an electroactive reporter that intercalates with hybridized DNA on the sensing site and is reduced at the cathodic pole. Luminol is oxidized on the anode, leading to an ECL signal that is directly correlated with the amount of short specific HIV oligonucleotide targets (dynamic range from 0.1 to $300 \mathrm{nM}$ ). A paper-based coupling between BPE and ECL was used for the rapid detection of $\mathrm{Hg}^{2+}$.(113) In this original strategy, the divalent cation mediates two thymine-enriched ssDNA probes to form a duplex where the ECL-active $\left[\mathrm{Ru}(\mathrm{phen})_{2} \mathrm{dppz}\right]^{2+}$ dye can readily intercalate. This organometallic complex features a dipyridophenazine (dppz) ligand which is responsible for the quenching of luminescence in aqueous media. However, the ECL signal is restored upon intercalation thanks to the local environment surrounding DNA. It is noteworthy that the same intercalator was also used directly for the detection of pathogenic bacteria.(48) Hydrophilic channels were waxscreen printed on filter paper, whereas the BE, as well as the two driving electrodes, were screen-printed into the channels by using a carbon ink. Polymerase chain reaction (PCR) amplification of the bacterial DNA was therefore detected by the ECL signal originating from the complex when intercalated into DNA.

Other strategies that take advantage of in-situ hybridization chain reactions (HCR) were also proposed. $(47,51)$ In a first example, luminol-modified Au NPs were immobilized at the surface of capture cancer cells via HCR with two hairpin DNA molecules (Figure 6).(47) The ECL coreactant is released from the target cells, leading to an ECL signal from luminol that is directly related to the number of cancer cells in the sample with a limit of detection of 40 cells per $\mathrm{mL}$. An HCR-induced ECL amplification strategy was also reported for the detection of DNA and $\mathrm{H}_{2} \mathrm{O}_{2}$.(51) The ECL signal that occurs at the anode of the BE is coupled with the electrochemical signal at the cathode, which is triggered by target DNA and amplified through the HCR process. 
The calibration curve shows a wide linear relationship between the strength of the ECL signal and the DNA concentration (from $0.1 \mathrm{nM}$ to $0.5 \mu \mathrm{M}$ ).

Recently, a smart area controllable interface was designed for the detection of tetracycline. It is based on a Janus glassy carbon bead, half covered by gold through BPE and functionalized with methylene blue (MB) and a DNA walker.(114) In the presence of tetracycline, an aptamer that is partially hybridized with the DNA walker is released from the surface to enable the formation of a new duplex with the walker and MB. The latter is cleaved from the surface with an endonuclease, which releases MB and triggers ECL detection in a closed-configuration.

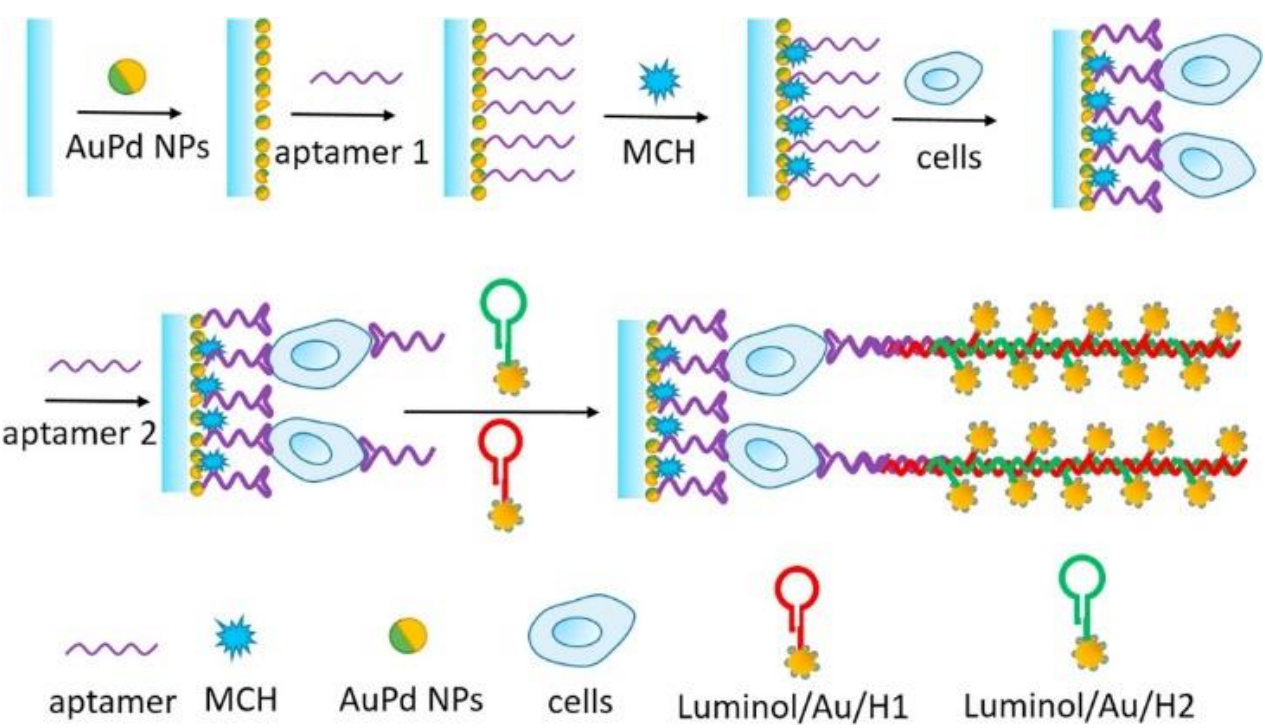

Figure 6 Illustration of a bottom up sandwich-type assembly process of a highly sensitive cytosensor (limit of detection of 40 cells $/ \mathrm{mL}$ ) developed in reference (47). Reproduced with permission from Elsevier.

\subsubsection{Antibodies}

Historically, ECL has been commercialized on the diagnostic market for the detection of specific antigens. Nowadays, the number of ECL-based immunoassays performed per year reaches almost two billion. While the vast majority of these tests are performed by using a conventional electrochemistry setup, BPE can offer an interesting alternative with many different experimental configurations.

$\mathrm{Li}$, Wang and coll. proposed a multichannel closed BPE array based on a polyethylene terephthalate (PET) membrane.(53) The multichannel PET membrane was etched in basic conditions before modification of the inner walls of the channels with Au nanofibers. This approach leads to an array of BEs with a controllable ion track density from $10^{4} / \mathrm{cm}^{2}$ up to 
$10^{8} / \mathrm{cm}^{2}$. It was combined with the $\left[\mathrm{Ru}(\mathrm{bpy})_{3}\right]^{2+} / \mathrm{TPA}$ system to generate the ECL output and applied to the detection of multiple targets including dopamine, $\alpha$-fetoprotein (AFP) and carcino-embryonic antigen (CEA). The same antigen target was also detected based on an electrochromic readout by the same authors.(72) The CEA aptamer was pre-anchored on the bipolar anode for capturing CEA through binding affinity, resulting in steric hindrance and lower current. Prussian Blue was used as an electrochromic indicator for reporting CEA concentration (typically in $\mathrm{ng} / \mathrm{mL}$ range) and distinguish samples of cancer patients from unaffected ones.

The ability of Ag@Au NPs to quench luminol ECL, as discussed above in section 2.3.2, was recently also exploited in the context of antibody based bipolar detection. This enables the measurement of PSA in a wide dynamic range from $0.1 \mu \mathrm{g} / \mathrm{mL}$ down to $0.1 \mathrm{pg} / \mathrm{mL}$, which fulfills the clinical requirements in PSA determination.(110) The possibility to apply this ECL test to real human serum samples was confirmed at a ng/mL PSA concentration and validated by comparing the results with a standard method. Another sensing platform for PSA monitoring in human blood serum was based on a multicolor ECL configuration in a closed bipolar setup.(67) This approach takes benefit of potential-resolved ECL, using a mixture of red and green luminophores $\left(\left[\mathrm{Ru}(\mathrm{bpy})_{3}\right]^{2+}\right.$ and $\operatorname{Ir}(\mathrm{ppy})_{3}$, respectively) with TPA as a co-reactant. The variation of interfacial potential at the poles of the BE results in an ECL color change and was used for the quantification of clinical biomarkers in a sandwiched antigen assay (Figure 7). With the same philosophy, a bidirectional ECL color switch was used for detecting multimarkers of prostate cancer.(37) This time, the authors selected an original cyclometalated complex of iridium, featuring difluoro-phenylpyridine (df-ppy) and picolinate (pic) ligands. The bidirectional color change from blue to red could be observed either with an increase or a decrease of applied voltage, due to the dual excitation properties of $\operatorname{Ir}(\mathrm{df}-\mathrm{ppy})_{2}$ (pic). This ECL switch was applied to the detection of three biomarkers of prostate cancer such as PSA, $\mu$ RNA and sarcosine. Finally, spatially resolved ECL ratiometry was adapted to detect PSA by using another ECL dye, namely graphite-like carbon nitride $\left(\mathrm{g}-\mathrm{C}_{3} \mathrm{~N}_{4}\right) .(115)$ The cathode of the closed $\mathrm{BE}$ is modified with $\mathrm{Au} @ \mathrm{~g}-\mathrm{C}_{3} \mathrm{~N}_{4}$ (blue emitter) which is coupled with the anodic ECL emission of $\left[\mathrm{Ru}(\mathrm{bpy})_{3}\right]^{2+}$. The corresponding PSA biosensor has a calibration curve with a linear range from 1 to $200 \mathrm{ng} / \mathrm{mL}$ and the measurements are in good agreement with the reference method used for clinical diagnostic.

In an effort towards parallel screening of samples, a very simple but efficient closed BE array was developed. The authors used an array of polymer-made wells that can be addressed individually. The proof-of-principle was achieved with an array composed of seven sensing 
compartments connected with a single reporting one.(52) The ECL reporting was achieved by using $\left[\mathrm{Ru}(\mathrm{bpy})_{3}\right]^{2+}$ dye together with ammonium oxalate coreactant. This sensing platform was applied to the detection of CEA biomarker with bimetallic Pd/Pt nanocrystals and a classic primary antibody/antigen/secondary antibody sandwich immunoassay. An impressive limit of detection down to $0.001 \mathrm{ng} / \mathrm{mL}$ was readily achieved.

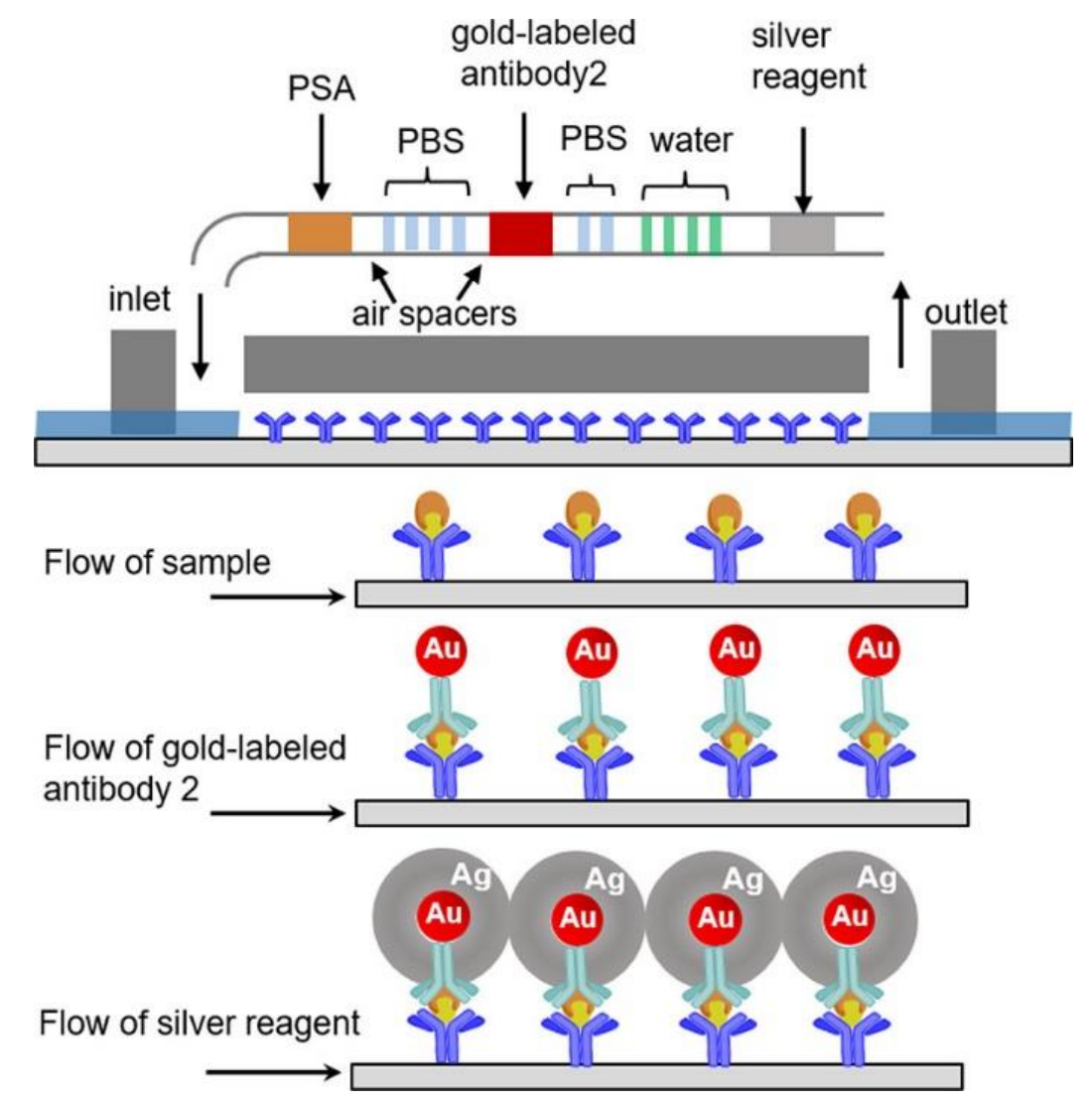

Figure 7 Stepwise injection of multiple (bio)chemical reagents into the BPE cell for the immunoassay reported in reference (67). Reproduced with permission from the American Chemical Society.

\subsubsection{Cells}

The progressive improvement and miniaturization of BPE's concepts and techniques allow now also to tackle more challenging biological entities such as living cells. Consequently BPE has not only been applied to the detection and imaging of cancer cells, $(38,40,78,115)$, but also to the on-chip monitoring of oxygen consumption by cell aggregates,(41) and to cell capture and lysis. $(43,116) \mathrm{Xu}$ and coworkers developed first a dual-BE array chip generating $\left[\mathrm{Ru}(\mathrm{bpy})_{3}\right]^{2+} \mathrm{ECL}$ and/or luminol ECL in two separated reservoirs.(38) In the presence of DNAzyme and $\mathrm{H}_{2} \mathrm{O}_{2}$, orange ECL emission decreased due to the quenching effect of $\mathrm{H}_{2} \mathrm{O}_{2}$, 
whereas blue ECL emission appears. The resulting ratiometric sensing principle provides better sensitivity and allows distinguishing cancer cells from normal ones because the normal cells produce less $\mathrm{H}_{2} \mathrm{O}_{2}$ and G-4 DNAzyme than cancer cells. The sensitivity was further improved by combining anodic dissolution and ECL detection on a similar bipolar sensing platform.(111) This analytical approach shows a very low detection limit down to 5 cells $/ \mathrm{cm}^{2}$. Cell metabolism was also monitored by designing a closed BPE device containing both analytical and reporter chambers.(41) Dioxygen consumption by cell aggregates at the cathodic poles of BPE arrays was converted to ECL signals at the anodic poles. The authors exploited the advantages of a closed configuration to detect many samples without cell damage. The reported analytical approach may be used for high-throughput assays of cell aggregates, in the frame of drug screening and cell differentiation.
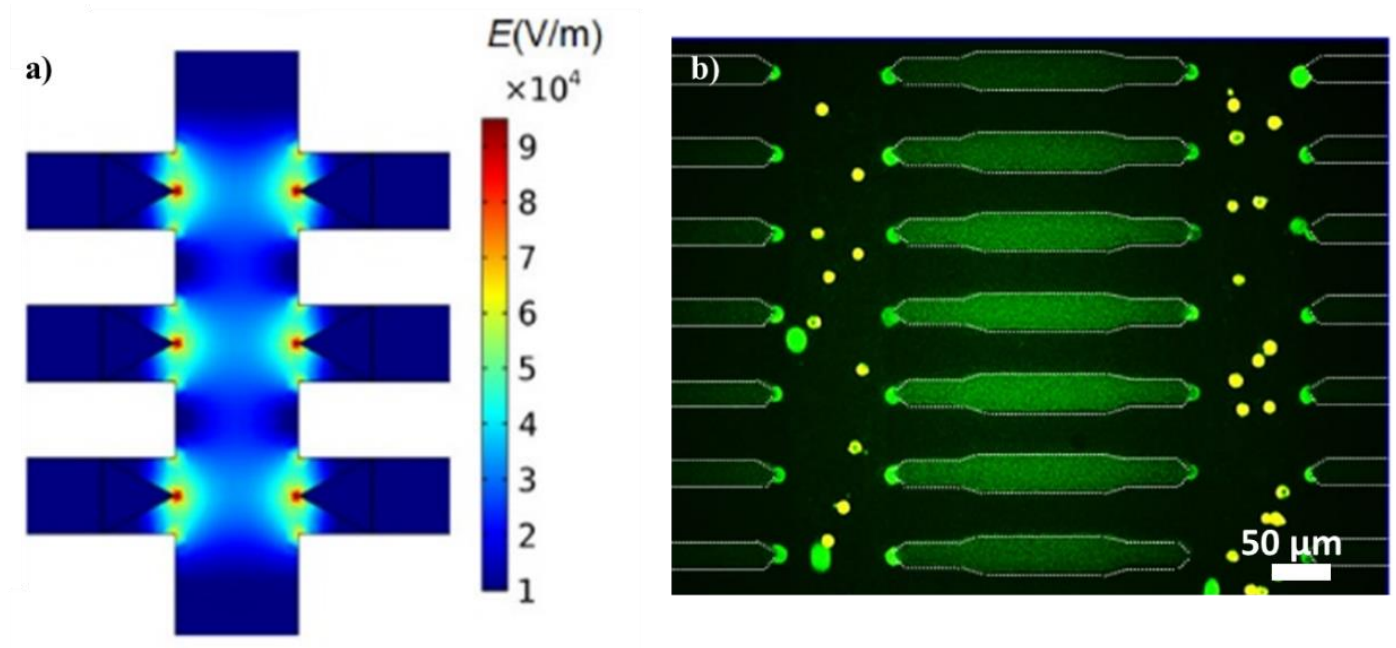

Figure 8 a) 2D simulation of the electric field distribution across a single microchannel flanked by 6 BE tips. b) Fluorescent image showing the capture of single MDA-MB-231 cells (green) in the presence of Jurkat cells (yellow) by applying an AC field using a BE array. Reprinted from reference (116) with permission from the American Chemical Society .

In addition to purely analytical applications, selective high-throughput single-cell capture based on dielectrophoresis has been reported using BPE.(116) Among cell manipulation methods, dielectrophoresis provides specific advantages such as improved selectivity, high cell viability and antibody-independent separation. The isolation of cells is based on their dielectric properties, which interact with an electric field gradient to create a movement. Anand and coworkers designed and fabricated an original dielectrophoresis device by integrating BEs.(116) They demonstrated the wireless control of the distribution of an AC 
electric field at a BE array to capture specifically single cells. The developed BE arrays impose an AC field across insulating barriers such as channel walls, which provokes the simultaneous capture of cells across parallel microchannels. The wireless feature enables very flexible device architectures with branched microchannels, increasing the throughput by offering 1408 potential capture sites. The reported high-throughput parallel-channel device was made of 32 parallel microchannels and each channel had 22 pockets extruded at each side. The corresponding 1408 micropockets provide discrete capture sites with defined volume, thus enabling single cell capture (Figure 8). Only two feeder electrodes were used to create the dielectrophoresis effect in an array format. This approach was further improved by a combination with parallel electrical cell lysis in a single microfluidic platform.(43) The specific capture of individual cells at such an array of wireless electrodes is a powerful technique and opens up promising possibilities to perform electrochemical analysis on the captured cells.

\section{Conclusion and outlook}

In this review article, we presented a selection of the most recent progress concerning the use of BPE in the frame of analytical chemistry. Completely new and very original approaches have emerged over the last few years. The considerable increase of the number of studies using BPE for an analytical purpose can be explained by several important advantages of BPE compared to classic electrochemistry:

1. BPE can be performed without a physical contact between the bipolar object and the power supply. The wireless nature of the experiments allows a straightforward miniaturization and an easy integration of BPE in microfluidic structures, which is especially interesting in the context of designing efficient analytical systems e.g. sophisticated lab-on-chip devices. Furthermore, this feature gives access to experiments which are difficult or completely impossible to carry out with a more classic electrochemical set-up.

2. As a direct consequence of the previous point, the technique allows addressing in parallel thousands or millions of objects with just a single pair of feeder electrodes. Naturally, this immediately opens up a whole avenue for many new types of experiments, especially when thinking, for example, about high throughput screening of analytes or (electro)catalytic features of materials and particles. 
3. BPE can be considered as a true low-cost technique, which in most cases only needs very simple instrumentation that can be easily implemented in every laboratory and handled even by inexperienced students.(117)

The direct consequence of these advantages is that the technique attracts a growing number of users as can be seen from the histogram in Figure 9, tracing the cumulative number of publications containing the keyword "bipolar electrochemistry" in the title or the abstract.

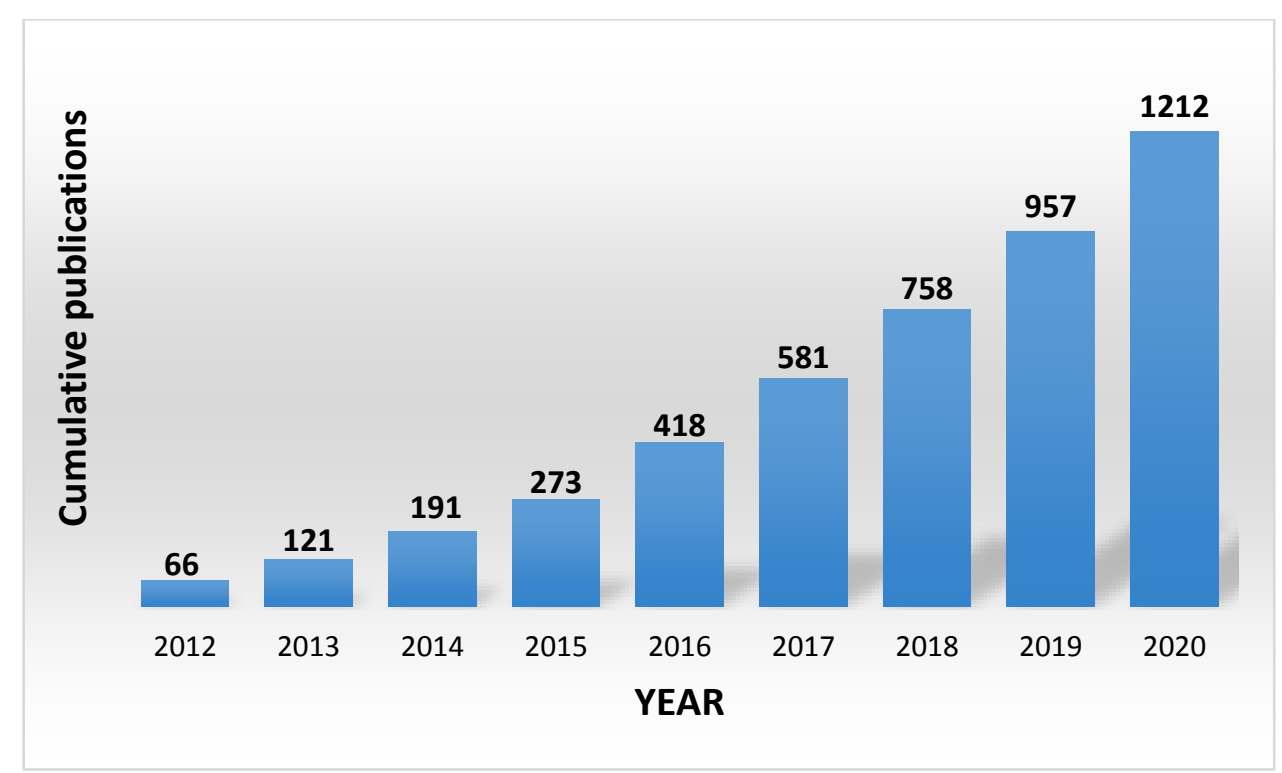

Figure 9 Histogram of the evolution of the cumulative number of publications containing the keyword "bipolar electrochemistry" in the title or the abstract (Source Google Scholar, Aug. 2020)

Obviously, these publications not only concern analytical chemistry, but also many other areas for which BPE is an attractive concept, ranging from materials science to catalysis and from energy storage/transformation to biology. However, it appears that studies in which analytical chemistry is the main focus of the work take an increasing portion in the last few years. We expect that this trend will still continue and we hope that the present review article might contribute to stimulating new ideas and motivate other scientists to integrate some aspects of BPE in their own work in order to drive the topic into so-far unexplored and sometimes completely unexpected directions.

\section{Acknowledgments}


This contribution has been funded by the European Research Council (ERC) under the

European Union's Horizon 2020 research and innovation program (grant agreement no.

741251, ERC Advanced Grant ELECTRA)

\section{References}

1. Bard AJ, Faulkner LR. 2001. Electrochemical methods: Fundamentals and Applications, 2nd ed.; John Wiley \& Sons, Inc

2. Hiddleston JN, Douglad AF. 1968. Fluidized Bed Electrodes- Fundamental Measurements and Implications. Nature 218: 601-02

3. van der Heiden CM, Raats SM, Boon JF. 1978. Fluidised Bed Electrolysis for Removal or Recovery of Metals from Dilute Solutions. Chem. Ind. 1: 465-68

4. Fleischmann M, Goodridge F, King CJH. 1978, US patent 4124453

5. Loget G, Zigah D, Bouffier L, Sojic N, Kuhn A. 2013. Bipolar Electrochemistry: From Materials Science to Motion and Beyond. Accounts of Chemical Research 46: 2513-23

6. Fosdick Stephen E, Knust Kyle N, Scida K, Crooks Richard M. 2013. Bipolar Electrochemistry. Angewandte Chemie International Edition 52: 10438-56

7. Shida N, Zhou Y, Inagi S. 2019. Bipolar Electrochemistry: A Powerful Tool for Electrifying Functional Material Synthesis. Accounts of Chemical Research 52: 2598-608

8. Koefoed L, Pedersen SU, Daasbjerg K. 2017. Bipolar electrochemistry-A wireless approach for electrode reactions. Current Opinion in Electrochemistry 2: 13-17

9. Sequeira CAC, Cardoso DSP, Gameiro MLF. 2016. Bipolar Electrochemistry, a Focal Point of Future Research. Chemical Engineering Communications 203: 1001-08

10. Loget G, Kuhn A. 2011. Shaping and exploring the micro- and nanoworld using bipolar electrochemistry. Analytical and Bioanalytical Chemistry 400: 1691-704

11. Karimian N, Hashemi P, Afkhami A, Bagheri H. 2019. The principles of bipolar electrochemistry and its electroanalysis applications. Current Opinion in Electrochemistry 17: 30-37

12. Pébère N, Vivier V. 2016. Local Electrochemical Measurements in Bipolar Experiments for Corrosion Studies. ChemElectroChem 3: 415-21

13. Munktell S, Tydén M, Högström J, Nyholm L, Björefors F. 2013. Bipolar electrochemistry for high-throughput corrosion screening. Electrochemistry Communications 34: 274-77

14. Zhou Y, Engelberg DL. 2020. Fast testing of ambient temperature pitting corrosion in type 2205 duplex stainless steel by bipolar electrochemistry experiments. Electrochemistry Communications 117: 106779

15. Ndungu PG. 2004. The Use of Bipolar Electrochemistry in Nanoscience: Contact Free Methods for the Site Selective Modification of Nanostructured Carbon Materials. PhD thesis, Drexel University, Philadelphia, PA

16. Guerrette JP, Oja SM, Zhang B. 2012. Coupled Electrochemical Reactions at Bipolar Microelectrodes and Nanoelectrodes. Analytical Chemistry 84: 1609-16

17. Cox JT, Guerrette JP, Zhang B. 2012. Steady-State Voltammetry of a Microelectrode in a Closed Bipolar Cell. Analytical Chemistry 84: 8797-804

18. Wood M, Zhang B. 2015. A Bipolar Electrochemical Method for Dynamic In Situ Control of Single Metal Nanowire Growth. ACS Nano 9: 2454-64

19. Zhang X, Li J, Jia X, Li D, Wang E. 2014. Full-Featured Electrochemiluminescence Sensing Platform Based on the Multichannel Closed Bipolar System. Analytical Chemistry 86: 5595-99

20. Comninellis C, Plattner E, Bolomey P. 1991. Estimation of current bypass in a bipolar electrode stack from current-potential curves. Journal of Applied Electrochemistry 21: 415-18

21. Hänni W, Perret A, Comninellis C. 2001, US patent 6306270 B1 
22. Sudoh M, Kodera T, Hino H, Shimamura H. 1988. Effect of anodic and cathodic reactions on oxidative degradation of phenol in an undivided bipolar electrolyser. Journal of Chemical Engineering of Japan 21: 198-203

23. Alkire RC, Engelmaier W, Kessler TJ. 1977, US patent 4043891

24. Kusakabe K, Morooka S, Kato Y. 1982. Current paths and electrolysis efficiency in bipolar packed-bed electrodes. Journal of Chemical Engineering of Japan 15: 45-50

25. Sentic M, Arbault S, Bouffier L, Manojlovic D, Kuhn A, Sojic N. 2015. 3D electrogenerated chemiluminescence: from surface-confined reactions to bulk emission. Chemical Science 6: 4433-37

26. Ordeig O, Godino N, del Campo J, Muñoz FX, Nikolajeff F, Nyholm L. 2008. On-Chip Electric Field Driven Electrochemical Detection Using a Poly(dimethylsiloxane) Microchannel with Gold Microband Electrodes. Analytical Chemistry 80: 3622-32

27. Warakulwit C, Nguyen T, Majimel J, Delville M-H, Lapeyre V, et al. 2008. Dissymmetric Carbon Nanotubes by Bipolar Electrochemistry. Nano Letters 8: 500-04

28. Bouffier L, Sojic N, Kuhn A. 2017. Capillary-assisted bipolar electrochemistry: A focused mini review. Electrophoresis 38: 2687-94

29. Fattah Z, Garrigue P, Goudeau B, Lapeyre V, Kuhn A, Bouffier L. 2013. Capillary Electrophoresis as a Production Tool for Asymmetric Microhybrids. Electrophoresis 34: 198590

30. de Poulpiquet A, Diez-Buitrago B, Dumont Milutinovic M, Sentic M, Arbault S, et al. 2016. Dual Enzymatic Detection by Bulk Electrogenerated Chemiluminescence. Analytical Chemistry 88: 6585-92

31. de Poulpiquet A, Diez-Buitrago B, Milutinovic M, Goudeau B, Bouffier L, et al. 2016. DualColor Electrogenerated Chemiluminescence from Dispersions of Conductive Microbeads Addressed by Bipolar Electrochemistry. ChemElectroChem 3: 404-09

32. Loget G, Kuhn A. 2011, Patent No. PCT/FR2011/053001

33. Bouffier L, Arbault S, Kuhn A, Sojic N. 2016. Generation of electrochemiluminescence at bipolar electrodes: concepts and applications. Analytical and Bioanalytical Chemistry 408: 7003-11

34. Li H-N, Yang D, Liu A-X, Liu G-H, Shan Y-P, et al. 2019. Facile Fabrication of Gold Functionalized Nanopipette for Nanoscale Electrochemistry and Surface Enhanced Raman Spectroscopy. Chinese Journal of Analytical Chemistry 47: e19104-e12

35. Gao R, Ying Y-L, Li Y-J, Hu Y-X, Yu R-J, et al. 2018. A 30 nm Nanopore Electrode: Facile Fabrication and Direct Insights into the Intrinsic Feature of Single Nanoparticle Collisions. Angewandte Chemie International Edition 57: 1011-15

36. Wang Y, Jin R, Sojic N, Jiang D, Chen H-Y. 2020. Intracellular Wireless Analysis of Single Cells by Bipolar Electrochemiluminescence Confined in Nanopipette. Angewandte Chemie International Edition 59: 10416-20

37. Wang Y-Z, Ji S-Y, Xu H-Y, Zhao W, Xu J-J, Chen H-Y. 2018. Bidirectional Electrochemiluminescence Color Switch: An Application in Detecting Multimarkers of Prostate Cancer. Analytical Chemistry 90: 3570-75

38. Zhang H-R, Wang Y-Z, Zhao W, Xu J-J, Chen H-Y. 2016. Visual Color-Switch Electrochemiluminescence Biosensing of Cancer Cell Based on Multichannel Bipolar Electrode Chip. Analytical Chemistry 88: 2884-90

39. Xiao Y, Xu L, Qi L-W. 2017. Electrochemiluminescence bipolar electrode array for the multiplexed detection of glucose, lactate and choline based on a versatile enzymatic approach. Talanta 165: 577-83

40. Wu M-S, Liu Z, Xu J-J, Chen H-Y. 2016. Highly Specific Electrochemiluminescence Detection of Cancer Cells with a Closed Bipolar Electrode. ChemElectroChem 3: 429-35

41. Ino K, Yaegaki R, Hiramoto K, Nashimoto Y, Shiku H. 2020. Closed Bipolar Electrode Array for On-Chip Analysis of Cellular Respiration by Cell Aggregates. ACS Sensors 5: 740-45 
42. Rahn KL, Rhoades TD, Anand RK. 2020. Alternating Current Voltammetry at a Bipolar Electrode with Smartphone Luminescence Imaging for Point-of-Need Sensing.

ChemElectroChem 7: 1172-81

43. Li M, Anand RK. 2019. Integration of marker-free selection of single cells at a wireless electrode array with parallel fluidic isolation and electrical lysis. Chemical Science 10: 1506-13

44. Eden A, Scida K, Arroyo-Currás N, Eijkel JCT, Meinhart CD, Pennathur S. 2019. Modeling Faradaic Reactions and Electrokinetic Phenomena at a Nanochannel-Confined Bipolar Electrode. The Journal of Physical Chemistry C 123: 5353-64

45. Zhang X, Zhai Q, Xu L, Li J, Wang E. 2016. Paper-based electrochemiluminescence bipolar conductivity sensing mechanism: A critical supplement for the bipolar system. Journal of Electroanalytical Chemistry 781: 15-19

46. Chen L, Zhang C, Xing D. 2016. Paper-based bipolar electrode-electrochemiluminescence (BPE-ECL) device with battery energy supply and smartphone read-out: $A$ handheld $E C L$ system for biochemical analysis at the point-of-care level. Sensors and Actuators B: Chemical 237: 308-17

47. Ge S, Zhao J, Wang S, Lan F, Yan M, Yu J. 2018. Ultrasensitive electrochemiluminescence assay of tumor cells and evaluation of $\mathrm{H}_{2} \mathrm{O}_{2}$ on a paper-based closed-bipolar electrode by insitu hybridization chain reaction amplification. Biosensors and Bioelectronics 102: 411-17

48. Liu H, Zhou X, Liu W, Yang X, Xing D. 2016. Paper-Based Bipolar Electrode Electrochemiluminescence Switch for Label-Free and Sensitive Genetic Detection of Pathogenic Bacteria. Analytical Chemistry 88: 10191-97

49. Lu H-J, Zhao W, Xu J-J, Chen H-Y. 2018. Visual electrochemiluminescence ratiometry on bipolar electrode for bioanalysis. Biosensors and Bioelectronics 102: 624-30

50. Zhang X, Bao N, Luo X, Ding S-N. 2018. Patchy gold coated $\mathrm{Fe}_{3} \mathrm{O}_{4}$ nanospheres with enhanced catalytic activity applied for paper-based bipolar electrode-electrochemiluminescence aptasensors. Biosensors and Bioelectronics 114: 44-51

51. Wu M, Xu N, Qiao J, Chen J, Jin L. 2019. Bipolar electrode-electrochemiluminescence (ECL) biosensor based on a hybridization chain reaction. Analyst 144: 4633-38

52. Ding S-N, Wang X-Y, Lu W-X. 2019. Switches-controlled bipolar electrode electrochemiluminescence arrays for high-throughput detection of cancer biomarkers. Journal of Electroanalytical Chemistry 844: 99-104

53. Zhai Q, Zhang X, Han Y, Zhai J, Li J, Wang E. 2016. A Nanoscale Multichannel Closed Bipolar Electrode Array for Electrochemiluminescence Sensing Platform. Analytical Chemistry 88: 945-51

54. Ongaro M, Gambirasi A, Ugo P. 2016. Closed Bipolar Electrochemistry for the Low-Potential Asymmetrical Functionalization of Micro- and Nanowires. ChemElectroChem 3: 450-56

55. Anderson TJ, Defnet PA, Zhang B. 2020. Electrochemiluminescence (ECL)-Based Electrochemical Imaging Using a Massive Array of Bipolar Ultramicroelectrodes. Analytical Chemistry 92: 6748-55

56. Chen J, Zhang J, Qiao J, Wu M. 2020. Closed bipolar electrochemical biosensor based on ohmic loss mechanism for noncontact measurements. Journal of Electroanalytical Chemistry 860: 113873

57. Stefano JS, Conzuelo F, Masa J, Munoz RAA, Schuhmann W. 2020. Coupling electrochemistry with a fluorescence reporting reaction enabled by bipolar electrochemistry. Journal of Electroanalytical Chemistry: 113921

58. Loget G, Fabre B. 2016. Light-Driven Bipolar Electrochemical Logic Gates with Electrical or Optical Outputs. ChemElectroChem 3: 366-71

59. Moghaddam MR, Carrara S, Hogan CF. 2019. Multi-colour bipolar electrochemiluminescence for heavy metal ion detection. Chemical Communications 55: 1024-27

60. Oja SM, Zhang B. 2016. Electrogenerated Chemiluminescence Reporting on Closed Bipolar Microelectrodes and the Influence of Electrode Size. ChemElectroChem 3: 457-64 
61. Defnet PA, Zhang B. 2020. Detection of Transient Nanoparticle Collision Events Using Electrochemiluminescence on a Closed Bipolar Microelectrode. ChemElectroChem 7: 252-59

62. Santos CS, Conzuelo F, Eßmann V, Bertotti M, Schuhmann W. 2019. Enhanced sensitivity of scanning bipolar electrochemical microscopy for $\mathrm{O}_{2}$ detection. Analytica Chimica Acta 1087: 36-43

63. Gamero-Quijano A, Herzog G, Scanlon MD. 2020. Aqueous surface chemistry of gold mesh electrodes in a closed bipolar electrochemical cell. Electrochimica Acta 330: 135328

64. Zhao W, Ma Y, Ye J, Jin J. 2020. A closed bipolar electrochemiluminescence sensing platform based on quantum dots: A practical solution for biochemical analysis and detection. Sensors and Actuators B: Chemical 311: 127930

65. Gamero-Quijano A, Molina-Osorio AF, Peljo P, Scanlon MD. 2019. Closed bipolar electrochemistry in a four-electrode configuration. Physical Chemistry Chemical Physics 21: 9627-40

66. Gao W, Muzyka K, Ma X, Lou B, Xu G. 2018. A single-electrode electrochemical system for multiplex electrochemiluminescence analysis based on a resistance induced potential difference. Chemical Science 9: 3911-16

67. Wang Y-Z, Xu C-H, Zhao W, Guan Q-Y, Chen H-Y, Xu J-J. 2017. Bipolar Electrode Based Multicolor Electrochemiluminescence Biosensor. Analytical Chemistry 89: 8050-56

68. Li H, Bouffier L, Arbault S, Kuhn A, Hogan CF, Sojic N. 2017. Spatially-resolved multicolor bipolar electrochemiluminescence. Electrochemistry Communications 77: 10-13

69. Scida K, Eden A, Arroyo-Currás N, MacKenzie S, Satik Y, et al. 2019. Fluorescence-Based Observation of Transient Electrochemical and Electrokinetic Effects at Nanoconfined Bipolar Electrodes. ACS Applied Materials \& Interfaces 11: 13777-86

70. Xu W, Ma C, Bohn PW. 2016. Coupling of Independent Electrochemical Reactions and Fluorescence at Closed Bipolar Interdigitated Electrode Arrays. ChemElectroChem 3: 422-28

71. Crouch GM, Oh C, Fu K, Bohn PW. 2019. Tunable optical metamaterial-based sensors enabled by closed bipolar electrochemistry. Analyst 144: 6240-46

72. Zhai Q, Zhang X, Xia Y, Li J, Wang E. 2016. Electrochromic sensing platform based on steric hindrance effects for CEA detection. Analyst 141: 3985-88

73. Xu W, Fu K, Ma C, Bohn PW. 2016. Closed bipolar electrode-enabled dual-cell electrochromic detectors for chemical sensing. Analyst 141: 6018-24

74. Jansod S, Cherubini T, Soda Y, Bakker E. 2020. Optical Sensing with a Potentiometric Sensing Array by Prussian Blue Film Integrated Closed Bipolar Electrodes. Analytical Chemistry

75. Seo M, Yeon SY, Yun J, Chung TD. 2019. Nanoporous ITO implemented bipolar electrode sensor for enhanced electrochemiluminescence. Electrochimica Acta 314: 89-95

76. Yuan F, Qi L, Fereja TH, Snizhko DV, Liu Z, et al. 2018. Regenerable bipolar electrochemiluminescence device using glassy carbon bipolar electrode, stainless steel driving electrode and cold patch. Electrochimica Acta 262: 182-86

77. Dauphin AL, Akchach A, Voci S, Kuhn A, Xu G, et al. 2019. Tracking Magnetic Rotating Objects by Bipolar Electrochemiluminescence. The Journal of Physical Chemistry Letters 10: 5318-24

78. Cao J-T, Wang Y-L, Zhang J-J, Dong Y-X, Liu F-R, et al. 2018. Immuno-Electrochemiluminescent Imaging of a Single Cell Based on Functional Nanoprobes of Heterogeneous $\mathrm{Ru}(\mathrm{bpy})_{3}{ }^{2+} @ \mathrm{SiO}_{2} /$ Au Nanoparticles. Analytical Chemistry 90: 10334-39

79. Hao N, Lu J, Dai Z, Qian J, Zhang J, et al. 2019. Analysis of aqueous systems using all-inorganic perovskite $\mathrm{CsPbBr}_{3}$ quantum dots with stable electrochemiluminescence performance using a closed bipolar electrode. Electrochemistry Communications 108: 106559

80. Li H, Garrigue P, Bouffier L, Arbault S, Kuhn A, Sojic N. 2016. Double remote electrochemical addressing and optical readout of electrochemiluminescence at the tip of an optical fiber. Analyst 141: 4299-304

81. Ibañez D, Heras A, Colina A. 2017. Bipolar Spectroelectrochemistry. Analytical Chemistry 89: 3879-83 
82. Eßmann V, Barwe S, Masa J, Schuhmann W. 2016. Bipolar Electrochemistry for Concurrently Evaluating the Stability of Anode and Cathode Electrocatalysts and the Overall Cell Performance during Long-Term Water Electrolysis. Analytical Chemistry 88: 8835-40

83. Takano S, Inoue KY, Ikegawa M, Takahashi Y, Ino K, et al. 2016. Liquid-junction-free system for substitutional stripping voltammetry using a closed bipolar electrode system.

Electrochemistry Communications 66: 34-37

84. Zhang J-D, Zhao W-W, Xu J-J, Chen H-Y. 2016. Electrochemical behaviors in closed bipolar system with three-electrode driving mode. Journal of Electroanalytical Chemistry 781: 56-61

85. Zhang L, Gupta B, Goudeau B, Mano N, Kuhn A. 2018. Wireless Electromechanical Readout of Chemical Information. Journal of the American Chemical Society 140: 15501-06

86. Assavapanumat S, Gupta B, Salinas G, Goudeau B, Wattanakit C, Kuhn A. 2019. Chiral platinum-polypyrrole hybrid films as efficient enantioselective actuators. Chemical Communications 55: 10956-59

87. Arnaboldi S, Gupta B, Benincori T, Bonetti G, Cirilli R, Kuhn A. 2020. Absolute Chiral Recognition with Hybrid Wireless Electrochemical Actuators. Analytical Chemistry 92: 1004247

88. Naser-Sadrabadi A, Zare HR. 2019. A highly-sensitive electrocatalytic measurement of nitrate ions in soil and different fruit vegetables at the surface of palladium nanoparticles modified DVD using the open bipolar system. Microchemical Journal 148: 206-13

89. Han C, Hao R, Fan Y, Edwards MA, Gao H, Zhang B. 2019. Observing Transient Bipolar Electrochemical Coupling on Single Nanoparticles Translocating through a Nanopore. Langmuir 35: 7180-90

90. Gupta B, Goudeau B, Kuhn A. 2017. Wireless Electrochemical Actuation of Conducting Polymers. Angewandte Chemie International Edition 56: 14183-86

91. Gupta B, Goudeau B, Garrigue P, Kuhn A. 2018. Bipolar Conducting Polymer Crawlers Based on Triple Symmetry Breaking. Advanced Functional Materials 28: 1705825

92. Gupta B, Afonso MC, Zhang L, Ayela C, Garrigue P, et al. 2019. Wireless Coupling of Conducting Polymer Actuators with Light Emission. ChemPhysChem 20: 941-45

93. Arora A, Eijkel JCT, Morf WE, Manz A. 2001. A Wireless Electrochemiluminescence Detector Applied to Direct and Indirect Detection for Electrophoresis on a Microfabricated Glass Device. Analytical Chemistry 73: 3282-88

94. Bouffier L, Manojlovic D, Kuhn A, Sojic N. 2019. Advances in bipolar electrochemiluminescence for the detection of biorelevant molecular targets. Current Opinion in Electrochemistry 16: 28-34

95. Xu W, Fu K, Bohn PW. 2017. Electrochromic Sensor for Multiplex Detection of Metabolites Enabled by Closed Bipolar Electrode Coupling. ACS Sensors 2: 1020-26

96. Yu X, Liang J, Yang T, Gong M, Xi D, Liu H. 2018. A resettable and reprogrammable keypad lock based on electrochromic Prussian blue films and biocatalysis of immobilized glucose oxidase in a bipolar electrode system. Biosensors and Bioelectronics 99: 163-69

97. Liu Z, Qi W, Xu G. 2015. Recent advances in electrochemiluminescence. Chemical Society Reviews 44: 3117-42

98. Liu M, Liu R, Wang D, Liu C, Zhang C. 2016. A low-cost, ultraflexible cloth-based microfluidic device for wireless electrochemiluminescence application. Lab on a Chip 16: 2860-70

99. Xiong X, Li Y, Yuan W, Lu Y, Xiong X, et al. 2020. Screen printed bipolar electrode for sensitive electrochemiluminescence detection of aflatoxin B1 in agricultural products. Biosensors and Bioelectronics 150: 111873

100. Liu C, Wang D, Zhang C. 2018. A novel paperfluidic closed bipolar electrodeelectrochemiluminescence sensing platform: Potential for multiplex detection at crossingchannel closed bipolar electrodes. Sensors and Actuators B: Chemical 270: 341-52

101. Hao R, Fan Y, Zhang B. 2017. Imaging Dynamic Collision and Oxidation of Single Silver Nanoparticles at the Electrode/Solution Interface. Journal of the American Chemical Society 139: $12274-82$ 
102. Ying Y-L, Hu Y-X, Gao R, Yu R-J, Gu Z, et al. 2018. Asymmetric Nanopore Electrode-Based Amplification for Electron Transfer Imaging in Live Cells. Journal of the American Chemical Society 140: 5385-92

103. Ismail A, Voci S, Pham P, Leroy L, Maziz A, et al. 2019. Enhanced Bipolar Electrochemistry at Solid-State Micropores: Demonstration by Wireless Electrochemiluminescence Imaging. Analytical Chemistry 91: 8900-07

104. Gholami F, Navaee A, Salimi A, Ahmadi R, Korani A, Hallaj R. 2018. Direct Enzymatic Glucose $/ \mathrm{O}_{2}$ Biofuel Cell based on Poly-Thiophene Carboxylic Acid alongside Gold Nanostructures Substrates Derived through Bipolar Electrochemistry. Scientific Reports 8: 15103

105. Eßmann V, Zhao F, Hartmann V, Nowaczyk MM, Schuhmann W, Conzuelo F. 2017. In Operando Investigation of Electrical Coupling of Photosystem 1 and Photosystem 2 by Means of Bipolar Electrochemistry. Analytical Chemistry 89: 7160-65

106. Gamero-Quijano A, Herzog G, Scanlon MD. 2019. Bioelectrochemistry of Cytochrome c in a closed bipolar electrochemical cell. Electrochemistry Communications 109: 106600

107. Chow K-F, Mavré F, Crooks RM. 2008. Wireless Electrochemical DNA Microarray Sensor. Journal of the American Chemical Society 130: 7544-45

108. Mavré F, Anand RK, Laws DR, Chow K-F, Chang B-Y, et al. 2010. Bipolar Electrodes: A Useful Tool for Concentration, Separation, and Detection of Analytes in Microelectrochemical Systems. Analytical Chemistry 82: 8766-74

109. Zhang X, Zhai Q, Xing H, Li J, Wang E. 2017. Bipolar Electrodes with $100 \%$ Current Efficiency for Sensors. ACS Sensors 2: 320-26

110. Shi H-W, Zhao W, Liu Z, Liu X-C, Wu M-S, et al. 2016. Joint enhancement strategy applied in ECL biosensor based on closed bipolar electrodes for the detection of PSA. Talanta 154: 16974

111. Shi H-W, Zhao W, Liu Z, Liu X-C, Xu J-J, Chen H-Y. 2016. Temporal Sensing Platform Based on Bipolar Electrode for the Ultrasensitive Detection of Cancer Cells. Analytical Chemistry 88: 8795-801

112. Poorghasem R, Saberi RS, Shayan M, Mehrgardi MA, Kiani A. 2016. Closed Bipolar Electrochemistry for the Detection of Human Immunodeficiency Virus Short Oligonucleotide. Electrochimica Acta 222: 1483-90

113. Liu H, Zhou X, Shen J, Xing D. 2017. Sensitive Detection of $\mathrm{Hg}^{2+}$ with Switchable Electrochemiluminescence Luminophore and Disposable Bipolar Electrode. ChemElectroChem 4: 1681-85

114. Jin L, Qiao J, Chen J, Xu N, Wu M. 2020. Combination of area controllable sensing surface and bipolar electrode-electrochemiluminescence approach for the detection of tetracycline. Talanta 208: 120404

115. Wang Y-Z, Zhao W, Dai P-P, Lu H-J, Xu J-J, et al. 2016. Spatial-resolved electrochemiluminescence ratiometry based on bipolar electrode for bioanalysis. Biosensors and Bioelectronics 86: 683-89

116. Li M, Anand RK. 2017. High-Throughput Selective Capture of Single Circulating Tumor Cells by Dielectrophoresis at a Wireless Electrode Array. Journal of the American Chemical Society 139: 8950-59

117. Zigah D. 2020. Le courant passe-épisode 3, https://www.youtube.com/watch?v=bifEBSB1 9Y. 